\title{
12-Week Behavioral Lifestyle Modification Program Increases Blood Antioxidant Enzyme Activities in Obese Adults
}

\author{
Md Rizman Md Lazin @ Md Lazim ${ }^{1}$,Rohana Abdul Jalil ${ }^{2}$ and Rahimah Zakaria ${ }^{1, *}$ \\ ${ }^{I}$ Department of Physiology, ${ }^{2}$ Department of Community Medicine, School of Medical Sciences, Universiti Sains \\ Malaysia, 16150 Kota Bharu, Kelantan, Malaysia
}

\begin{abstract}
This study aims to evaluate the outcome of a 12-week behavioral lifestyle modification program focusing on the blood oxidative stress levels/activities in obese subjects and aims to examine the association between the changes in blood oxidative stress levels/activities and modifications in serum lipid profile concentrations. An interventional study was conducted involving thirty-four obese adults, all of whom were recruited from the Obesity Clinic, Hospital Universiti Sains Malaysia. The subjects participated in the 12-week behavioral lifestyle modification program consisting of diet and exercise interventions. Blood was obtained from the subjects at baseline (week 0) and after 12 weeks (week 13) for the determination of oxidative stress levels/activities and lipid profile concentrations. Plasma glutathione peroxidase (GPx) activity and 4-hydroxynonenal (4-HNE) level increased significantly, and the ratio of reduced glutathione (GSH) to oxidized glutathione (GSSG) was decreased following 12-week interventions. Other enzymatic antioxidant activities such as catalase (CAT) and superoxide dismutase (SOD) were increased, but the changes were not statistically significant. Changes in plasma GPx activity were negatively correlated with changes in serum triglyceride (TG) and VLDL-cholesterol concentrations. In conclusion, diet and exercise interventions in the 12-week behavioral lifestyle modification program were effective in increasing antioxidant enzyme activities in obese adults.
\end{abstract}

Keywords: Obesity, antioxidant, lipid profile, lifestyle modification, diet, exercise.

\section{INTRODUCTION}

A strong association between systemic oxidative stress and adiposity has been reported in human beings [1-3]. Furukawa et al. [4] put forward an imperative mechanism of obesity-related cardiovascular disease through elevated oxidative stress in accumulated body fat. Abnormal lipid profiles such as hypercholesterolemia stimulate the production of superoxide anion radicals from the smooth muscle cells of vessels, an event that leads to increased oxidation of LDL (oxLDL) [5]. The ox-LDL is mostly taken up by macrophage scavenger receptors, rather than the normal LDL receptor pathway, thus inducing atherosclerosis [6]. Additionally, the increased production of reactive oxygen species reduces the production and consequently the bioavailability of nitric oxide (NO), leading to vasoconstriction, platelet aggregation and adhesion of neutrophils to the endothelium [5]. All these events are known to contribute to the development of atherosclerosis.

The behavioral lifestyle modification program for weight loss has beneficial effects regarding the prevention of cardiovascular disease $[7,8]$. Most of the program consists of either exercise or dietary changes alone or a combination of both interventions which thus effectively reduce body weight, body fat and oxidative stress levels after a short

*Address correspondence to this author at the Department of Physiology, School of Medical Sciences, Universiti Sains Malaysia, 16150 Kubang Kerian, Kelantan, Malaysia. Tel/Fax: 609-7676156/609-7653370;

E-mail: rahimah@kck.usm.my period of time [9-11]. Exercise training is associated with a modest but significant reduction in fat mass [12] as well as oxidative stress $[13,14]$. Dietary modification in the weight loss program was conducted on prepubertal obese subjects [15] and obese hypertensive adults [16] reduced body mass index (BMI) and improved oxidative stress. A 21-day residential rigorous dietary and exercise intervention program profoundly reduced $\mathrm{BMI}$ and increased the antioxidant-oxidant balance [7, 17]. A recent randomized, controlled trial conducted on 173 overweight or obese, postmenopausal, sedentary women were subjected to aerobic exercise intervention and the outcome revealed a decrease in oxidative stress with only minimal changes to their body mass or body composition [18].

Therefore, the present study aims to evaluate the effect of a 12-week behavioral lifestyle modification program on blood oxidative stress levels/activities in obese subjects and to examine the association between the changes in blood oxidative stress levels/activities and the changes in serum lipid concentrations. In the present study, only the main antioxidant enzymes such as catalase (CAT) activity, glutathione peroxidase (GPx) activity and superoxide dismutase (SOD) level, and a major product of lipid peroxidation i.e. 4-hydroxynonenal (4-HNE) were measured.

\section{MATERIALS AND METHODOLOGY}

\section{Study Subjects}

Patients attending the Obesity Clinic Hospital Universiti Sains Malaysia were invited to voluntarily participate in this behavioral lifestyle modification program which consisted of 
dietary and exercise interventions. A total of 34 eligible subjects aged between 18-62 years were recruited. Only 29 out of 34 subjects (85\%) successfully completed this study. The reason for subjects dropping out of the study was noncompliance to the program schedule. All the subjects were obese with a BMI $>30 \mathrm{~kg} / \mathrm{m}^{2}$. Subjects were excluded if they were pregnant or had the intention of getting pregnant during the intervention period and if they had enrolled within the past three months in a formal weight reduction program or clinical trial or had uncontrolled hypertension, diabetes, or other serious illnesses during the previous 6 months.

\section{Behavioral Lifestyle Modification Program}

The research design was approved by the Research and Ethics Committee of Universiti Sains Malaysia. The protocol as well as the potential risks and benefits of participating in the program were explained to each subject before he/she gave written consent.

Once enrolled in the program, subjects underwent a complete medical history and physical examinations to ensure that they were fit for the exercise intervention. A diary was given to each subject and they were asked to selfmonitor and record their daily food intake and physical activities. Fasting blood samples were obtained from all the subjects for the determination of oxidative stress levels/activities and lipid and lipoproteins concentrations at pre-intervention (week 0) and post-intervention (week 13).

About $8 \mathrm{ml}$ of fasting venous blood was obtained from each subject and the blood was collected into EDTA and plain containers. The blood in the EDTA container was transported in ice to prevent blood clotting and centrifuged at $1000 \mathrm{~g}$ for 10 minutes at $4^{\circ} \mathrm{C}$ to obtain plasma. The blood in the plain container was allowed to clot at room temperature for $1 / 2$ an hour, and centrifuged at $2000 \mathrm{~g}$ for 15 minutes at $4^{\circ} \mathrm{C}$ to obtain serum. Both plasma and serum were aliquoted and stored at $-80^{\circ} \mathrm{C}$ until assayed.

Subjects were instructed to attend the weekly exercise program for 12 weeks which consisted of a brisk walk for 2$4 \mathrm{~km}$ (approximately 1 hour), 30 minutes aerobic exercise and 30 minutes dumbbell $(4 \mathrm{~kg})$ resistance exercise. Apart from the weekly program, subjects were instructed to perform daily moderate intensity physical activities such as, brisk walking at home etc. The subjects were requested to gradually increase the duration and frequency of their physical activity from 10 to 60 minutes each session, 3 to 5 days per week.

The weekly dietary program consisted of twelve nutrition education modules presented by nutritionists (second author). The program is designed to allow the subjects to eat ad labium without restriction on calorie (kcal/day) or food compositions. The subjects' food diary was discussed individually with the nutritionists. The subjects were advised to modify the type and/or amount of their usual food intake and whether the subject's dietary intake proved either insufficient or exceeded his/her requirements. If the subject's weekly weight loss goal was not achieved, they were instructed to discuss in a small group to devise a plan on how to achieve the target weight loss for the next visit.

\section{Determination of Blood Lipid Profile and Oxidative Stress Biomarkers}

Serum triglyceride (TG), VLDL-cholesterol, LDLcholesterol and HDL-cholesterol were quantified using a clinical chemistry analyzer (Abbott Architect c8000).

Plasma CAT, plasma GPx and serum SOD activities, blood ratio of reduced glutathione $(\mathrm{GSH})$ to oxidized glutathione (GSSG), and plasma 4-HNE concentration were determined using commercially available ELISA kits (Calbiochem, EMD Biosciences, Inc. for GSH:GSSG ratio, CAT, GPx and SOD activities; OxiSelect, Cell Biolabs, Inc. for 4-HNE) according to the manufacturer's instructions. Total protein was determined using a commercially available protein assay (Bio-Rad Laboratories, Inc.).

\section{Statistical Analysis}

The results were analyzed using the PASW Statistics version 18 software. A paired $t$ test was used to examine variance in the outcomes between pre- and post-intervention differences means of numerical variables. The Pearson correlation analysis was used to examine the association between changes in blood oxidative stress level/activities and changes in the plasma lipid profile. A probability values $<0.05$ was considered statistically significant.

\section{RESULTS}

Characteristics of all the subjects are shown in Table $\mathbf{1 .}$ After the diet and exercise interventions for 12 weeks, the body weight ( $88.7 \pm 14.5$ vs. $82.4 \pm 12.6 \mathrm{~kg})$, BMI (35.7 \pm 4.3 vs. $\left.33.2 \pm 3.6 \mathrm{~kg} / \mathrm{m}^{2}\right)$ and body fat mass $(43.2 \pm 6.8 \mathrm{vs}$. $40.5 \pm 6.9 \%$ ) were considerably reduced.

The mean serum lipid and lipoproteins concentrations at pre- and post-interventions are shown in Table 2 . There was a notable reduction in the mean serum TG and VLDLcholesterol concentrations when compared with pre- and post-interventions. However, there were no significant differences in the mean concentrations of total cholesterol, LDL-cholesterol and HDL-cholesterol when compared to pre- and post-interventions.

Levels/activities of blood oxidative stress biomarkers at pre- and post-interventions are presented in Table 3. Plasma GPx activity and 4-HNE level were significantly increased, and blood GSH/GSSG ratio was radically reduced after only 12 weeks of the intervention program. Other antioxidants enzymes, plasma CAT activity and serum SOD level were increased but were not statistically significant.

The scatter plots illustrate significant negative correlations between the changes in the plasma GPx activity with the changes in plasma TG $(r=-0.430, p<0.05)$ and VLDL-cholesterol $(r=-0.429, \mathrm{p}<0.05)$ Fig. $(\mathbf{1})$.

\section{DISCUSSION}

Most of the published data related to interventional studies either focusing on dietary, exercise, pharmacological, surgical or a combination of dietary and exercise interventions within the obese population measured biomarkers of oxidative damage such as malondialdehyde (MDA), ox-LDL, thiobarbituric acid reactive substances (TBARS), protein carbonyl, 8-hydroxy-2'-deoxyguanosine 
Table 1. Characteristics of the Study Subjects

\begin{tabular}{|c|c|c|c|}
\hline \multicolumn{2}{|c|}{ Characteristics of Subjects } & \multirow[t]{2}{*}{$\mathbf{N}(\%)$} & \multirow{2}{*}{$\begin{array}{c}\text { Mean } \pm \text { SD } \\
42.06 \pm 9.63\end{array}$} \\
\hline Age (years) & & & \\
\hline \multicolumn{4}{|l|}{ Sex } \\
\hline & Female & $25(86)$ & \\
\hline & Male & $4(14)$ & \\
\hline \multicolumn{4}{|c|}{ Educational level } \\
\hline & Primary & $2(7)$ & \\
\hline & Secondary & $16(55)$ & \\
\hline & Tertiary & $11(38)$ & \\
\hline \multicolumn{4}{|c|}{ Employment Status } \\
\hline & Employed & $20(69)$ & \\
\hline & Unemployed & $9(31)$ & \\
\hline \multicolumn{4}{|l|}{ Marital Status } \\
\hline & Married & $26(90)$ & \\
\hline & Single & $3(10)$ & \\
\hline
\end{tabular}

Values are frequency counts $(\%)$ or means \pm standard deviation. N, number of subjects.

Table 2. Serum Lipid and Lipoproteins Concentrations at Pre- and Post-Interventions

\begin{tabular}{|c|c|c|c|}
\hline & Pre-Intervention & Post-Intervention & Changes $(\Delta)$ \\
\hline \hline TG $(\mathrm{mmol} / \mathrm{l})$ & $2.05 \pm 1.93$ & $1.53 \pm 1.32^{*}$ & $-0.51 \pm 0.87$ \\
\hline Total C $(\mathrm{mmol} / \mathrm{l})$ & $5.94 \pm 1.03$ & $5.78 \pm 0.79$ & $-0.16 \pm 0.87$ \\
\hline VLDL-C $(\mathrm{mmol} / \mathrm{l})$ & $0.93 \pm 0.88$ & $0.70 \pm 0.60^{*}$ & $-0.23 \pm 0.39$ \\
\hline LDL-C $(\mathrm{mmol} / \mathrm{l})$ & $3.69 \pm 0.77$ & $3.82 \pm 0.80$ & $+0.13 \pm 0.72$ \\
\hline HDL-C $(\mathrm{mmol} / \mathrm{l})$ & $1.32 \pm 0.32$ & $1.26 \pm 0.31$ & $-0.06 \pm 0.19$ \\
\hline
\end{tabular}

Values are expressed as means \pm SD ( $\mathrm{n}=29$ subjects). TG, triglyceride; Total C, total cholesterol; VLDL-C, very low density lipoprotein-cholesterol; LDL-C, low density lipoproteincholesterol; HDL-C, high density lipoprotein-cholesterol. $* P<0.05$, after (post-intervention) versus before (pre-intervention).

Table 3. Levels/Activities of Blood Oxidative Stress Biomarkers at Pre- and Post-Interventions

\begin{tabular}{|c|c|c|c|}
\hline Markers & Pre-Intervention & Post-Intervention & $\boldsymbol{P}$ Value \\
\hline \hline Blood GSH/GSSG ratio & $24.85 \pm 26.49$ & $10.08 \pm 13.91$ & $0.032 *$ \\
\hline Plasma GPx $(\mathrm{nmol} / \mathrm{min} / \mathrm{ml})$ & $106.71 \pm 26.48$ & $128.51 \pm 35.14$ & $0.015^{*}$ \\
\hline Plasma HNE $(\mu \mathrm{g} / \mathrm{ml})$ & $3.98 \pm 2.90$ & $5.71 \pm 3.38$ & $0.016^{*}$ \\
\hline Serum SOD $(\mathrm{U} / \mathrm{ml})$ & $0.09 \pm 0.04$ & $0.11 \pm 0.04$ & 0.231 \\
\hline Plasma CAT $(\mathrm{nmol} / \mathrm{min} / \mathrm{ml})$ & $56.76 \pm 23.97$ & $58.90 \pm 15.28$ & 0.698 \\
\hline
\end{tabular}

Values are expressed as mean $\pm \mathrm{SD}$ ( $\mathrm{n}=29$ subjects). GSH/GSSG, ratio of reduced to oxidized glutathione; GPx, glutathione peroxidase; 4-HNE, 4-hydroxynonenal; CAT, catalase; SOD, superoxide dismutase. $* P<0.05$, after (post-intervention) versus before (pre-intervention).

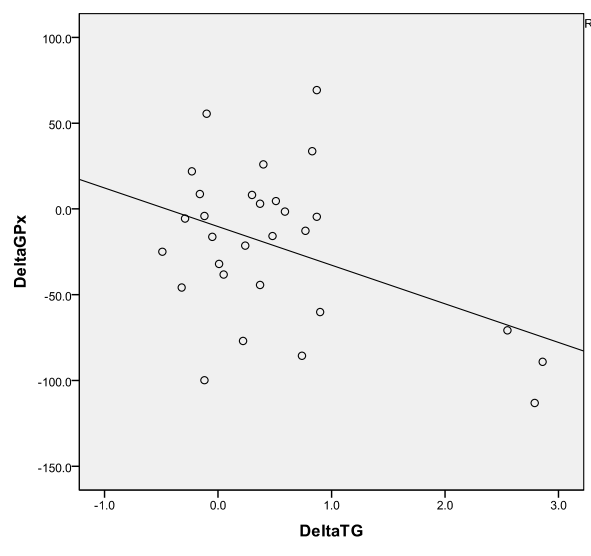

A

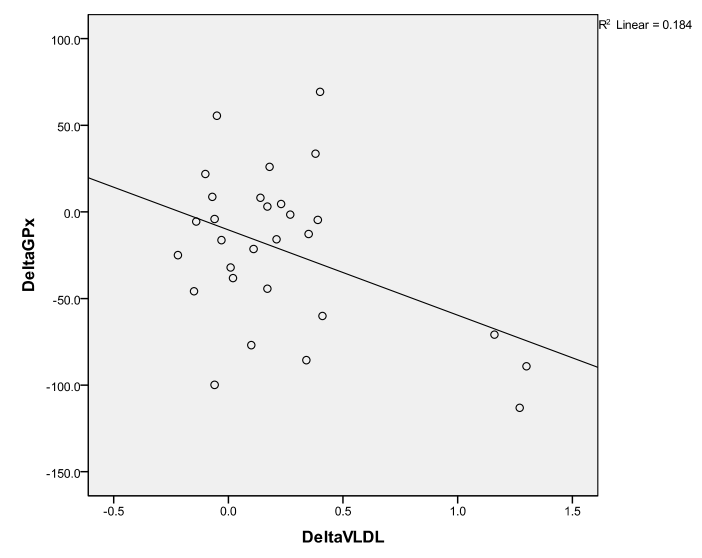

B

Fig. (1). Scatter plots illustrate correlations between the changes in plasma GPx activity and the changes in (A) TG and (B) VLDL-cholesterol $(n=29$ subjects $)$. 
(8-OhDG) and 8-epi-prostaglandin $\mathrm{F}_{2 \alpha}\left(8\right.$-epi-PGF $\left.\mathrm{F}_{2 \alpha}\right)$ levels [19-22]. So far, a very limited number of studies measure the effect of these interventional studies on main antioxidant enzymes such as SOD, GPx and CAT or use more reliable oxidative damage markers to lipids such as $4-\mathrm{HNE}$ and $\mathrm{F}_{2}$ isoprostanes. Skrha et al. [23] measured SOD levels following dietary intervention in obese diabetic and nondiabetic subjects. They found that one week consisting of a very low calorie diet administration, decreased oxidative stress in obese non-diabetic subjects but only partly in diabetic persons [23]. Another study found that there were no favorable changes in the levels of SOD, CAT and total anti-oxidative capacity following a 16-week internetdelivered lifestyle physical activity intervention [Active Living Every Day-Internet (ALED-I)] in overweight adults [24]. The present study is the first evidence of behavioral lifestyle program involving a combination of diet and exercise interventions measuring the main oxidative stress biomarkers in obese adults.

The major limitation of this study was the relatively small sample size because of the need to run the 12-week intervention program in a small group of 12-15 participants due to close supervision. Dropout in the middle of the program was due to non compliance to weekly activities and this also contributed to the small sample size. As a result, the findings of this study needs to be interpreted cautiously and are not easily applicable to the entire obese population of obese adults. Nevertheless, this intervention study allows direct comparison of the levels/activities of oxidative stresses biomarkers between pre- (served as control) and post-interventions.

The increased trend in the levels/activities of antioxidant enzymes following diet and exercise interventions was noted in this study. However, only GPx level was increased significantly and this could be explained by the small sample size. It is not clear from this study whether exercise, diet or a combination of both interventions had a significant contribution to the increased antioxidant defense.

The challenge of studying free radical systems in vivo is the short half-life and low concentrations [25]. Thus, in this study, the blood GSH:GSSG assay was utilized to determine the oxidative stress status among subjects following the interventions. The blood GSH:GSSG ratio was considerably reduced and reflected an increased oxidative stress following the behavioral lifestyle program. The result was further confirmed by increased oxidative damage to lipids as shown by increased plasma 4-HNE level after intervention. The increased oxidative stress could be induced by the obese state [19], exercise [26, 27] or both.

The present study also demonstrated significant negative correlations between changes in plasma GPx activity and changes in plasma TG and VLDL-cholesterol concentrations. This finding suggests that the participants who had greater reduction in their plasma TG and VLDLcholesterol concentrations were associated with increased plasma GPx activity.

The increased oxidative stress or oxidative damage may serve as an essential "signal" for the up regulation in antioxidant defenses as seen in this study, thereby providing protection against subsequent exposure to pro-oxidant (free radicals) environments within susceptible individuals [28].

In conclusions, this study has proved that the 12-week behavioral lifestyle program improves antioxidant defense probably as a result of exercise-induced reactive oxygen and nitrogen species production. It is safe to speculate that longterm adherence to lifestyle modifications among obese subjects will reduce the risk of cardiovascular disease through an improvement in lipid profile and antioxidant defense.

\section{ACKNOWLEDGEMENTS}

This work was financially supported by Research University Grant 1001/PPSP/812031

\section{CONFLICT OF INTEREST}

The authors confirm that this article content has no conflicts of interest.

\section{REFERENCES}

[1] Olusi SO. Obesity is an independent risk factor for plasma lipid peroxidation and depletion of erythrocyte cytoprotectic enzymes in humans. Int J Obes Relat Metab Disord 2002; 26(9): 1159-64.

[2] Keaney JR JF, Larson MG, Vasan RS, et al. Obesity and systemic oxidative stress: clinical correlates of oxidative stress in the Framingham Study. Arterioscler Thromb Vasc Biol 2003; 23: 4349.

[3] Farshad A, Fereydoun S, Mahmoud D, Abbas RF. Assessment of antioxidant enzyme activities in erythrocytes of pre-hypertensive and hypertensive women. JRMS 2010; 15(5): 270-78.

[4] Furukawa S, Fujita T, Shimabukuro M, et al. Increased oxidative stress in obesity and its impact on metabolic syndrome. J Clin Invest 2004; 114(12): 1752-61.

[5] Vogiatzi G, Tousoulis D, Stefanadis C. The role of oxidative stress in atherosclerosis. Hellenic J Cardiol 2009; 50: 402-9.

[6] Van Gaal LF, Mertens IL, De Block CE. Mechanisms linking obesity with cardiovascular disease. Nature 2006; 444: 875-80.

[7] Roberts CK, Vaziri ND, Barnard RJ. Effect of diet and exercise intervention on blood pressure, insulin, oxidative stress, and nitric oxide availability. Circulation 2002; 106: 2530-32.

[8] Knoops KT, De Groot LC, Kromhout D, et al. Mediterranean diet, lifestyle factors, and 10-year mortality in elderly European men and women: the HALE project. JAMA 2004; 292: 1433-9.

[9] Dattilo AM, Kris-Etherton PM. Effects of weight reduction on blood lipids and lipoproteins: a meta-analysis. Am J Clin Nutr 1992; 56(2): 320-8.

[10] Van Gaal LF, Wauters MA, De Leeuw IH. The beneficial effects of modest weight loss on cardiovascular risk factors. Int J Obes Relat Metab Disord 1997; 21(Suppl1): S5-S9.

[11] Poobalan A, Aucott L, Smith WC, et al. Effects of weight loss in overweight/obese individuals and long-term lipid outcomes-a systematic review. Obes Rev 2004; 5: 43-50.

[12] Catenacci VA, Wyatt HR. The role of physical activity in producing and maintaining weight loss. Nat Clin Pract Endocrinol Metab 2007; 3(7): 518-29.

[13] Ozcelik O, Dogan H, Kelestimur H. Effects of eight weeks of exercise training and orlistat therapy on body composition and maximal exercise capacity in obese females. Public Health 2006; 120(1): 76-82.

[14] Marciniak A, Brzeszczyńska J, Gwoździński K, Jegier A. Antioxidant capacity and physical exercise. Biol Sport 2009; 26(3): 197-213.

[15] Mohn A, Catino M, Capanna R, Giannini C, Marcovecchio M, Chiarelli F. Increased oxidative stress in prepubertal severely obese children: effect of a dietary restriction-weight loss program. J Clin Endocrinol Metab 2005; 90: 2653-8.

[16] Lopez D, Orta X, Casos K, et al. Upregulation of endothelial nitric oxide synthase in rat aorta after ingestion of fish oil-rich diet. Am J Physiol Heart Circ Physiol 2004; 287: H567-72. 
[17] Roberts CK, Won D, Pruthi S, et al. Effect of a short-term diet and exercise intervention on oxidative stress, inflammation, MMP-9, and monocyte chemotatic activity in men with metabolic syndrome factors. J Appl Physiol 2006; 100: 1657-65.

[18] Campbell PT, Gross MD, Potter JD, et al. Effect of exercise on oxidative stress: A 12-month randomized, controlled trial. Med Sci Sports Exerc 2010; 42(8): 1448-53.

[19] Vincent HK, Innes KE, Vincent KR. Oxidative stress and potential interventions to reduce oxidative stress in overweight and obesity. Obesity 2007; 9: 813-39.

[20] Rector RS, Warner SO, Liu Y, et al. Exercise and diet induced weight loss improves measures of oxidative stress and insulin sensitivity in adults with characteristics of the metabolic syndrome Am J Physiol Endocrinol Metab 2007; 293: E500-6.

[21] Kelishadi R, Hashemi M, Mohammadifard N, et al. Association of changes in oxidative and proinflammatory states with changes in vascular function after a lifestyle modification trial among obese children. Clin Chem 2008; 54(1): 147-53.

[22] Wycherley TP, Brinkworth GD, Noakes M, et al. Effect of caloric restriction with and without exercise training on oxidative stress and endothelial function in obese subjects with type 2 diabetes. Diabetes Obes Metab 2008; 10: 1062-73.

[23] Skrha J, Kunesova M, Hilgertova J, et al. Short-term very low calorie diet reduces oxidative stress in obese type 2 diabetic patients. Physiol Res 2005; 54: 33-9.

[24] Smith DT, Carrl LJ, Dorozynskil C, et al. Internet-delivered lifestyle physical activity intervention: limited inflammation and antioxidant capacity efficacy in overweight adults. J Appl Physiol 2009; 106(1): 49-56.

[25] Nedeljkovic ZS, Gokce N. Oxidative stress and exercise. J Cardiopulm Rehabil Prev 2005; 25: 220-1.

[26] Alok KB, Amritlal M, Dipanjan C, Sajal C. Oxidant, antioxidant and physical exercise. Mol Cell Biochem 2003; 253: 307-12.

[27] Vincent HK, Bourguignon CM, Vincent KR, Weltman AL, Bryant M, Taylor AG. Antioxidant supplementation lowers exerciseinduced oxidative stress in young overweight adults. Obesity 2006; 14: 2224-35.

[28] Fisher-Wellman K, Bloomer RJ. Acute exercise and oxidative stress: a 30 year history. Dyn Med 2009; 8(1): 1-25.

Received: March 26, 2012

(C) Lazim et al.; Licensee Bentham Open.

This is an open access article licensed under the terms of the Creative Commons Attribution Non-Commercial License (http://creativecommons.org/licenses/by-nc/3.0/) which permits unrestricted, non-commercial use, distribution and reproduction in any medium, provided the work is properly cited. 